\title{
Relationship between Occupational and Physical Therapist Students' Belongingness and Perceived Competence in the Clinic using the Ascent to Competence Scale
}

Patti J. Berg-Poppe

University of South Dakota

Joy R. Karges

University of South Dakota

Ranelle Nissen

University of South Dakota

Susan Deutsch

LifeScape

Kendra Webster

Sundog Rehabilitation

Follow this and additional works at: https://encompass.eku.edu/jote

Part of the Education Commons, and the Occupational Therapy Commons

\section{Recommended Citation}

Berg-Poppe, P. J., Karges, J. R., Nissen, R., Deutsch, S., \& Webster, K. (2017). Relationship between Occupational and Physical Therapist Students' Belongingness and Perceived Competence in the Clinic using the Ascent to Competence Scale. Journal of Occupational Therapy Education, 1 (3). https://doi.org/10.26681/jote.2017.010303 


\title{
Relationship between Occupational and Physical Therapist Students' Belongingness and Perceived Competence in the Clinic using the Ascent to Competence Scale
}

\begin{abstract}
Clinical education experiences (CEEs) serve an essential role in physical therapist (PT) and occupational therapist (OT) student development. The Ascent to Competence Scale (ACS) measures valuable attributes of belongingness, competence, and welcoming associated with CEE placement. The purpose of this study was to examine the relationship between $\mathrm{PT}$ and OT students' belongingness and perceived competence during CEE using the ACS. A survey consisting of 35 questions from the ACS measuring students' feelings of belongingness and perceived competence in the clinic was administered to PT and OT students from 7 Midwest universities. Respondents rated statements using a 5-point Likert-type scale ("never true" to "always true"). Ascent to Competence items were aggregated to develop belongingness and perceived competence constructs. One hundred nineteen $(67.2 \%$ PT, 32.8\% OT) of 509 (23.4\% response) eligible students completed the survey. Results of a linear regression analysis showed belongingness in the clinical environment significantly predicted perceived competence measures, $F(1,117)=182.389 ; P=\mathrm{r}^{2}=.609, \mathrm{y}(\mathrm{comp})=$ $.721(\mathrm{Xbel})+1.249$. Cumulative weeks in $\mathrm{CEE}$ and practice environment did not contribute to the predictive model. The analysis lends further support to the role that belongingness plays in advancing perceived competence during the CEE. The results suggest that supportive clinical education environments can positively impact student learning by promoting a sense of belongingness among student therapists.
\end{abstract}

\section{Keywords}

Clinical education, Ascent to Competence Scale, belongingness, competence

\section{Creative Commons License (c) (i) (3)}

This work is licensed under a Creative Commons Attribution-Noncommercial-No Derivative Works 4.0 License. 


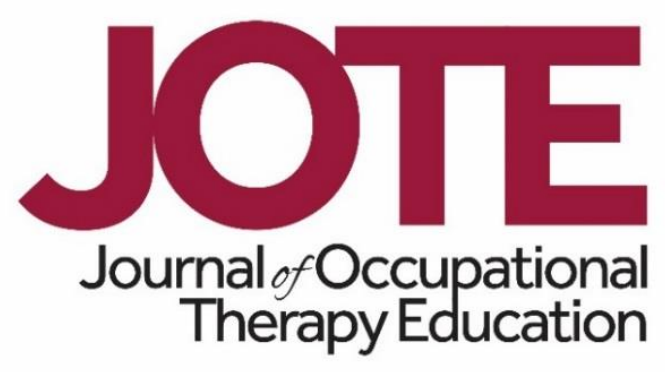

Volume 1, Issue 3

\begin{abstract}
Relationship between Occupational and Physical Therapist Students' Belongingness and Perceived Competence in the Clinic using the Ascent to Competence Scale
\end{abstract}
Patti Berg-Poppe, PT, PhD ${ }^{1}$, Joy Karges, PT, EdD ${ }^{1}$, Ranelle Nissen, OTR/L, PhD ${ }^{1}$, Susan Deutsch, PT, DPT ${ }^{2}$, and Kendra Webster, PT, DPT ${ }^{3}$ University of South Dakota ${ }^{1}$, LifeScape ${ }^{2}$, and Sundog Rehabilitation, LLC ${ }^{3}$ United States

\begin{abstract}
Clinical education experiences (CEEs) serve an essential role in physical therapist (PT) and occupational therapist (OT) student development. The Ascent to Competence Scale (ACS) measures valuable attributes of belongingness, competence, and welcoming associated with CEE placement. The purpose of this study was to examine the relationship between PT and OT students' belongingness and perceived competence during CEE using the ACS. A survey consisting of 35 questions from the ACS measuring students' feelings of belongingness and perceived competence in the clinic was administered to PT and OT students from 7 Midwest universities.

Respondents rated statements using a 5-point Likert-type scale ("never true" to "always true"). Ascent to Competence items were aggregated to develop belongingness and perceived competence constructs. One hundred nineteen (67.2\% PT, 32.8\% OT) of 509 (23.4\% response) eligible students completed the survey. Results of a linear regression analysis showed belongingness in the clinical environment significantly predicted perceived competence measures, $F(1,117)=182.389 ; P=<0.0001, r^{2}=.609, \mathrm{y}(\mathrm{comp})$ $=.721(\mathrm{Xbel})+1.249$. Cumulative weeks in CEE and practice environment did not contribute to the predictive model. The analysis lends further support to the role that belongingness plays in advancing perceived competence during the CEE. The results suggest that supportive clinical education environments can positively impact student learning by promoting a sense of belongingness among student therapists.
\end{abstract}




\section{INTRODUCTION}

The goal of the clinical education experience (CEE; a.k.a. fieldwork or clinical internship) is to develop occupational (OT) and physical (PT) therapists who are confident and competent practitioners ready to enter into the work environment. Clinical education is vitally important to students' education, as it provides professional interaction opportunities and hands-on learning. Successful mentorship by a clinical supervisor may utilize role modeling, opportunities for delivery of direct clinical services, and appropriate level of support for the student's proficiency level (AOTA, 2012) to guide the student toward clinical competence. For those with a stake in successful clinical education programming, it is important to determine and measure clinical site variables that facilitate learning. Thus, this study examined the relationship between student belongingness and perceived competence during clinical education.

\section{Competence}

Competence is "the ability to do something successfully or efficiently" (Oxford Dictionary, 2017). Development of student competence in the clinic requires the acquisition and mastery of key foundational skills and attributes. The American Occupational Therapy Association (AOTA) has identified the constructs of knowledge, critical reasoning, interpersonal skills, performance skills, and ethical practice as the core standards for continued competence in practice (AOTA, 2015). Students who demonstrate competence with these essential foundational standards are well on their way toward achieving entry-level clinical practice skills.

Students are assessed on their levels of achieved competence periodically throughout the CEE. Physical therapy students are formally assessed with the Clinical Performance Instrument (American Physical Therapy Association [APTA], 2006) at midterm and at final. Student PTs also self-assess their personal feelings of competence at midterm and final. Occupational therapy students are formally assessed, at minimum, twice throughout the CEE. The typical method of evaluation for the Level II fieldwork portion of the CEE is the AOTA Fieldwork Performance Evaluation (AOTA, 2002). This tool allows the fieldwork educator to formally assess the student at midterm and final. Students are encouraged to rate themselves on these same measures. The goal of assessment throughout the CEE is to provide formative feedback about the growth and development of skills such as clinical reasoning and the application of professional standards of practice.

However, while these formal assessments are an important gauge on whether a student is on-track to achieve entry-level clinical status, such formal evaluations are not comprehensive in their ability to fully detect student learning, nor do they inform about barriers and facilitators to student learning. Prior research indicates that learning is linked closely to external factors such as a positive educator attitude (Grenier, 2015), welcoming environment (Martin, Morris, Moore, Sadlo, \& Crouch, 2004), ability to be autonomous (Clarke, Martin, Sadlo, \& de-Visser, 2014), and a positive orientation period (Evenson, Barnes, \& Cohn, 2002). The student's formal evaluation score may not accurately reflect the student's potential if these positive external factors are not 
present. Supportive research asserts that the culture of the clinical learning environment is an essential characteristic in facilitating student learning. Cultures that facilitate student learning are ones that value student clinical education (Recker-Hughes et al., 2014; Rindflesch, Hoversten, Patterson, Thomas, \& Dunfee, 2013) and create a supportive climate in which students feel accepted and are afforded a degree of autonomy (Recker-Hughes et al., 2014). These facilitatory cultures for competence are one construct of clinical education. Another construct is the student's sense of belongingness.

\section{Belongingness}

Levett-Jones and Lathlean (2009) assert "the need to belong exerts a powerful influence on cognitive processes, emotional patterns, behavioural responses, health and wellbeing" (p. 2870). The construct of "belongingness" is operationalized as "the need for connectedness, acceptance, and fit, as well as the assurance of having a legitimate place in the clinical milieu" (Levett-Jones \& Lathlean, 2009, p. 2873). While it is argued that a sense of belonging creates furtive ground for learning, Levett-Jones and Lathlean argue that when this basic need is unsatisfied, the drive to achieve a sense of belongingness may also overthrow inquisitiveness in favor of acquiescence and, in such case, can interfere with learning.

A model of learning in nurse education created by Edgecombe and Bowden (2009) identified factors that affect a student's development into proficient novice registered nurses. One of the subthemes of this model is the construct of belonging. A positive or negative sense of belonging impacted the student nurse education for all participants in the study. Students reported that a sense of trust and value communicated by facility staff had a positive impact on their learning experience. Conversely, a sense of isolation associated with student status negatively impacted the learning experience (Edgecombe \& Bowden, 2009). These findings are supported by the Ascent to Competence Framework (ACF), a conceptual model developed by Levett-Jones and Lathlean (2009).

\section{Ascent to Competence Conceptual Framework}

Maslow's pyramid of basic needs proposes that certain basic physiological and psychological needs must be met in order for a human to both survive and thrive. These hierarchical levels, in order of ascension, are physiological, safety, love and belonging, esteem, and self-actualization. Higher-level needs are not achieved without first securing lower level needs (Maslow, 1987). A mixed-model approach was undertaken by Levett-Jones and colleagues (Levett-Jones \& Lathlean, 2009; Levett-Jones, Lathlean, Higgins, \& McMillan, 2009) to explore the experience of belongingness as it relates to Maslow's hierarchy of needs (Maslow, 1987). The researchers conceptualized that belongingness, as a basic psychological need, would be foundational to students achieving their potential for hierarchically superior achievements. Such achievements include clinical competence, which parallels Maslow's "self-actualization" (Maslow, 1987). In fact, the qualitative work affirmed that a sense of belongingness supports engagement, motivates meaningful involvement, and promotes an essential feeling that 
one is an integral member of the clinical team. The ACF is a theoretical model grounded in Maslow's hierarchy of needs theory and proposes that student clinical development is reliant upon the hierarchical acquisition of ascending basic needs (Levett-Jones \& Lathlean, 2009). Each clinical placement opportunity offers a degree of potential, whether large or small, to cultivate safety and security, a sense of belongingness, and self-concept in the student learner and to shape the learning experience toward the achievement of practice competency (Levett-Jones \& Lathlean, 2009).

During the CEE, the degree to which a student experiences belongingness may influence not only a student's capacity to learn but also the motivation to engage in clinical learning opportunities and other learning events (Levett-Jones et al., 2009). In fact, Nolan (1998) determined the degree of acceptance and belongingness students felt while completing a CEE was directly related to their active participation and learning during the experience.

Feelings of alienation and negative staff-student relationships lead some students to report overall negative CEEs (Levett-Jones et al., 2009). Nursing literature reports a link between the conceptual levels of belongingness and perceived competence in the clinical education setting. However, this link has not been researched or studied in the fields of OT and PT. Because the CEE is so important to the development of competent OTs and PTs, research in this area is essential in assuring the highest level of learning is achieved by students (Levett-Jones et al., 2009).

\section{Purpose}

The purpose of this study was to examine the relationships between OT and PT students' belongingness and perceived competence in the clinic using the Ascent to Competence Scale [ACS] (McCoy, Levett-Jones, \& Pitt, 2013). The specific objective of this study was to investigate the relationship between students' feelings of belongingness and perceived competence within the clinical setting. The researchers hypothesized that a direct positive relationship would exist between students' feelings of belongingness in the clinical setting and their perceived level of learning and competency.

\section{METHODOLOGY}

\section{Design}

The study employed a correlational design and surveyed OT and PT students from entry-level educational programs within participating Midwestern institutions.

\section{Subjects}

Study inclusion requirements included enrollment in a participating university's OT or PT graduate program and current enrollment in a CEE. The surveyed sample $(87.4 \%$ female) was drawn from seven participating Midwest universities. All participating programs had a minimum CEE length of six weeks; maximum length varied by program curricular need. This variation in CEE length was used to justify a data collection week 
that captured all participants. For that reason, exclusion criteria included those who were participating in a CEE less than five weeks in length.

The sample of convenience consisted of 119 of 509 (23.4\% response) eligible entry level (Masters) OT (32.8\%) and (Doctoral) PT (67.2\%) students. Mean age of students was $24.7(S D=2.9$ ) years (see Table 1; see Figure 1). Cumulative weeks enrolled in CEE for participants ranged from 5 weeks to 23 weeks, with the mean clinical experience being $14.9(S D=6.3$ ) weeks (see Table 1). Responding students reported placement within a variety of practice settings (see Table 2 ).

Table 1

Demographic Characteristics by Student Discipline

\begin{tabular}{cccc}
\hline & $\begin{array}{c}\text { Occupational } \\
\text { Therapy Students } \\
\text { (OTS; } \mathbf{n = 3 9 )}\end{array}$ & $\begin{array}{c}\text { Student Physical } \\
\text { Therapists } \\
\text { (SPT; } \mathbf{n = 8 0 )}\end{array}$ & $\begin{array}{c}\text { Sample } \\
\text { (N=119) }\end{array}$ \\
\hline Cumulative Weeks Full-time CEE & & \\
\hline Minimum & 5 weeks & 5 weeks & \\
Maximum & 20 weeks & 23 weeks & $14.91,6.28$ \\
Mean, $S D$ & $19.28,2.43$ & $12.78,6.49$ & \\
\hline Age in Years & & $24.75,2.83$ & $24.66,2.87$ \\
\hline Mean, $S D$ & $24.46,3.00$ &
\end{tabular}

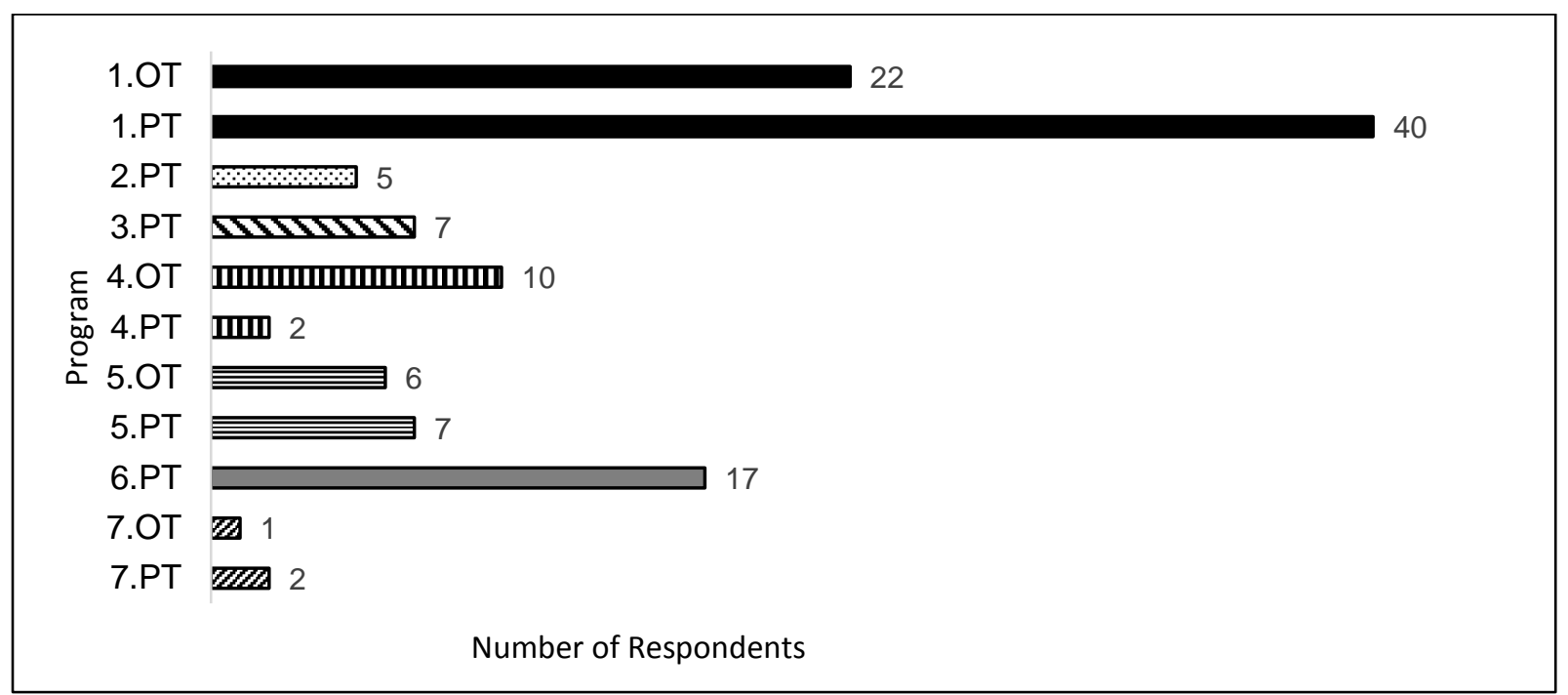

Figure 1. Number of respondents by program. 
Table 2

Student Distribution: Primary Practice Setting of Clinical Education Experience

\begin{tabular}{|c|c|c|}
\hline & Frequency & Percent \\
\hline General Practice & 22 & $18.49 \%$ \\
\hline Inpatient Acute & 26 & $21.85 \%$ \\
\hline Inpatient Rehabilitation & 9 & $7.56 \%$ \\
\hline Inpatient Subacute/Long-term Care/Extended Care Facility & 16 & $13.45 \%$ \\
\hline Mental Health & 2 & $1.68 \%$ \\
\hline Outpatient Orthopedic & 30 & $25.21 \%$ \\
\hline Outpatient Neurologic & 3 & $2.52 \%$ \\
\hline Pediatrics: Medical (Inpatient or Outpatient) & 6 & $5.04 \%$ \\
\hline Pediatrics: Educational (School-Based, Behavioral, or Early Childhood) & 4 & $3.36 \%$ \\
\hline Other & 1 & $0.84 \%$ \\
\hline Total & 119 & $100.00 \%$ \\
\hline \multicolumn{3}{|c|}{$\begin{array}{l}\text { Instrument } \\
\text { The ACS (McCoy et al., 2013) was developed specifically for purposes of measuring } \\
\text { student feelings of belongingness and perceived competence during the CEE. The ACS } \\
\text { (McCoy et al., 2013) comprises } 35 \text { statements related to and measuring students' } \\
\text { feelings of being welcomed ( } 6 \text { items; i.e., "I was introduced to the therapy staff in the } \\
\text { clinic/unit when I commenced the placement"), belongingness ( } 22 \text { items; i.e., "I } \\
\text { developed a good working relationship with my mentor"), and perceived competence } \\
\text { (13 items; i.e., "I felt more capable at the end of my placement") in the clinical education } \\
\text { process. McCoy, Levett-Jones, and Pitt (2013) showed evidence of ACS construct } \\
\text { validity through exploratory factor analysis with promax oblique rotation; the results } \\
\text { yielded a three component structure with each subscale demonstrating high internal } \\
\text { consistency (being welcomed, } \alpha=0.89 ; \text { belongingness, } \alpha=0.90 ; \text { perceived } \\
\text { competence, } \alpha=0.93 \text { ). The full scale's internal consistency was determined using } \\
\text { Cronbach's coefficient alpha, with satisfactory reliability ( } \alpha=0.98 \text { ). Questions were } \\
\text { answered using a 5-point rating scale, with } 1=\text { never true, } 2=\text { rarely true, } 3=\text { sometimes } \\
\text { true, } 4=\text { often true and } 5=\text { always true. Reverse scoring was used for the scale's } 3 \\
\text { negative items. Permission to use the ACS with modifications specific to the PT and OT } \\
\text { disciplines was received from the instrument authors (personal e-mail communication, } \\
\text { April } 17,2013 \text { ). The modified ACS was disseminated via SurveyMonkey® } \\
\text { (https://www.surveymonkey.com/) and included questions related to cumulative number } \\
\text { of weeks of full-time CEE across-settings, age at the start of current CEE, rural or urban } \\
\text { environment, practice setting, and therapy discipline. }\end{array}$} \\
\hline
\end{tabular}




\section{Procedure}

The research study was approved by the institution's IRB prior to survey dissemination, and all participants provided consent. Data were collected from a sample of convenience, consisting of students in OT and PT programs from participating institutions. The research institution's Academic Fieldwork Coordinator (AFWC) and Director of Clinical Education (DCE) communicated directly with the participating universities' DCEs/AFWCs, who subsequently forwarded all communication to their students. Other than the students from the research partner's university, there was no direct communication between the research partners and the student participants. Clinical placements between programs differed in length, location, and timing within the curriculum (see Figure 2). Researchers communicated with the DCEs/AFWCs at Weeks 1,4 , and 5 of the chosen CEE's. Initial information was forwarded to students during Week 1 of the CEE. A reminder email with the electronic survey link was forwarded during Week 4 indicating the survey would be open during the fifth week of the CEE. Students were alerted during the middle of Week 5 that the survey would close Friday of the same week.

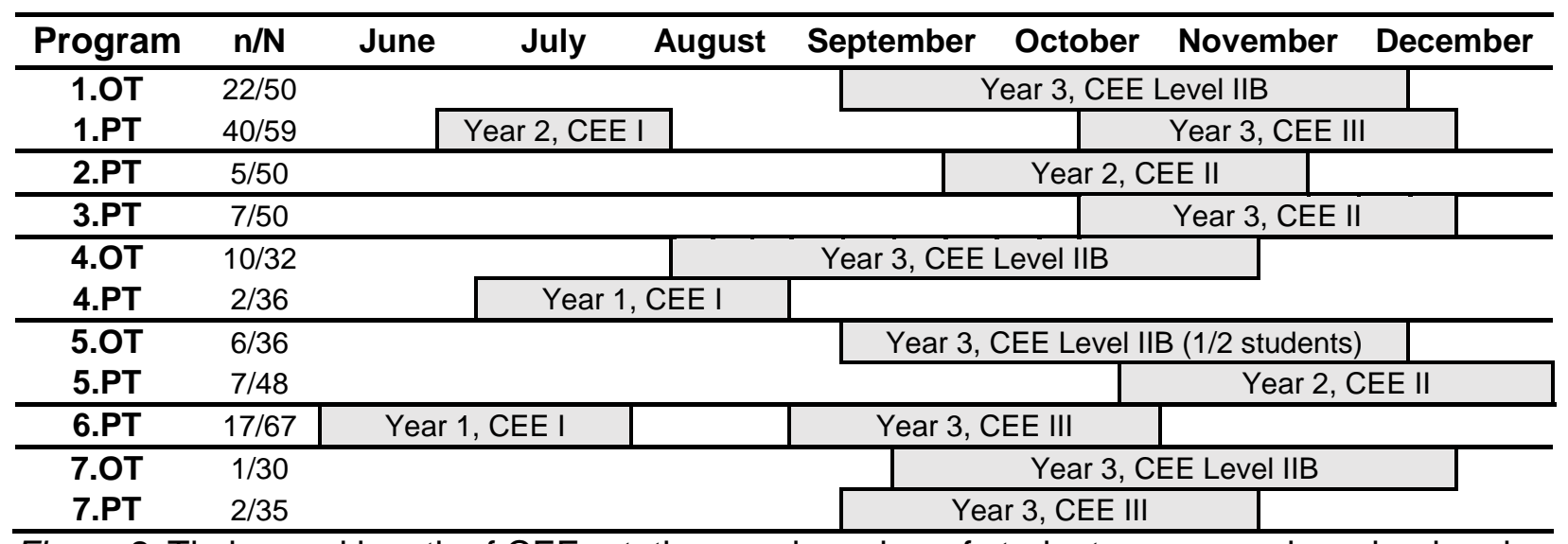

Figure 2. Timing and length of CEE rotations and number of student responses by school and discipline.

\section{Data Analysis}

Statistical analysis was conducted using descriptive statistics for demographic information. Questions from main survey components were aggregated to develop belongingness and perceived competence constructs. Linear regression was used to analyze the relationship between belongingness, experience as measured by cumulative weeks of CEE, type of setting, and perceived competence. Statistical analysis was completed using IBM SPSS Statistics for Windows (Version 24.0) with alpha set at 0.05 . 


\section{RESULTS}

A stepwise linear regression was conducted with the "perceived competence" variable regressed on predictors, "belongingness," cumulative weeks of CEE (as an indicator of experience within clinical environments), and primary setting (to account for varied caseload complexities that may be setting-specific). Cumulative weeks of CEE and primary setting did not significantly contribute to a predictive model (see Table 3). The best fit model showed feelings of belongingness in the clinical environment significantly predicted perceived competence, $F(1,117)=182.389 ; p=<0.0001, r^{2}=0.609$, $y_{\text {comp }}=$ $0.721 \mathrm{xbel}+1.249$. Thus, $60.9 \%$ of the variance in the perceived competence within the clinical environment was predicted by belongingness, indicating a large effect.

Table 3

Stepwise Regression Model Predicting Perceived Learning and Competence

\begin{tabular}{lccc}
\hline Dependent Variable & $\boldsymbol{\beta}$ & $\mathbf{t}$ & $\boldsymbol{p}$ \\
\hline Belongingness & 0.713 & 13.066 & $0.0001^{\mathrm{a}}$ \\
Cumulative Weeks in Full-time CEE & -0.003 & -0.720 & 0.473 \\
Primary Setting Type & -0.006 & -0.444 & 0.658 \\
${ }^{\mathrm{a} p}<<0.0001$ & &
\end{tabular}

To study the influence of gender and OT or PT discipline on the relationship between belongingness and perceived competence, an examination of moderation using a series of regression analyses was undertaken. Gender moderated the relationship between belongingness and perceived competence $\left(R^{2}=0.622, R^{2}\right.$ change $\left.=0.013, p=0.046\right)$, with a stronger direct association between belongingness and perceived competence reported by male student therapists $(r=0.906, p=<0.0001)$ than female student therapists $(r=0.767 . p=<0.0001)$. Discipline did not influence the relationship between belongingness and perceived competence.

\section{DISCUSSION}

Student healthcare professionals involved in the clinical education process are often surrounded with competing needs, such as those of security and acceptance, connectedness and respect, and freedom to self-direct. The extent to which these needs are met is decisive in determining whether a student placed within a particular CEE is likely to ascend the ACF hierarchy toward successful student learning. The purpose of this study was to investigate the relationship between students' feelings of belongingness and perceived competence within the clinical setting. The current study concludes that when students feel a stronger sense of belongingness, they are more apt to have higher levels of perceived competence. This relationship is not OT or PT discipline-specific, and transcends differences in practice environments. 
The results support research conducted by several in the field of nursing (Grobecker, 2016; Kim \& Park, 2011; Levett-Jones \& Lathlean, 2009; Luanaigh, 2015; Russell, Alliex, \& Gluyas, 2016; Thomson, Docherty, \& Duffy, 2017) and indicate that the level of perceived belongingness that students feel during CEE is a significant predictor of perceived competence. A path model generated by Kim and Park (Kim \& Park, 2011) helps explain the relationship between belongingness and competence observed in the current research. Their model, accounting for $37 \%$ of the variation for self-directed learning, revealed that reported level of belongingness within the CEE had a negative effect on stress and positive effect on satisfaction, self-esteem, and self-directed learning. Belongingness had a direct effect on self-directed learning as well as an indirect effect on self-directed learning through self-esteem. In fact, while belongingness and self-esteem had a positive effect on self-directed learning, the model revealed no effects of stress and satisfaction on self-directed learning. Drawing from this study, it may be speculated that students in the current study who felt a stronger sense of belonging benefitted from the direct effects of belongingness as well as the indirect effects through self-esteem buoyed by a supportive clinical environment. Future research may test this path model with a sample of OT and PT students, examining the relationships among belongingness, satisfaction, self-directed learning, self-esteem, and stress.

The results also indicate that the relationship between competence and belonging was more strongly relevant for male students than for female students. The reasoning for this difference is not clearly understood; however, a study by Kim and Sax (2009) found similar gender differences. Their study examined the effect of student-faculty interaction as mediated by several student characteristics, one of which was gender. The results of that study indicate the male students had a higher sense of belonging when they had increased levels of interaction with faculty regarding course related content. Females also benefited from course-related interaction; however, they preferred a one on one context, whereas males were more comfortable in the group context.

One study that may give some explanation to why male students feel a greater sense of belonging with more direct interaction involves the field of science. A study conducted by Moss-Racusin, Dovidio, Brescoll, Graham and Handelsman (2012) found that male students were ranked as more competent and hirable with a higher starting pay offer and increased mentoring by faculty as compared to an identical female student. The results of the study indicate the differences were likely due to inadvertent gender biases held by faculty regardless of the faculty's gender, age, rank, or field (Moss-Racusin, Dovidio, Brescoll, Graham, \& Handelsman, 2012). This study could explain an unintentional bias by CEE supervisors toward male students, resulting in a higher sense of belonging, but this is pure speculation. Understanding the full context for which a gender difference exists during CEE is beyond the scope of this current study and is an area for future research. 


\section{Limitations}

Limitations of this study included a small sample size, questionable generalizability, selfreported data, and survey distribution timing. The study included participants from multiple programs and graduate universities, which enhanced the generalizability of the results. However, the relatively low response rate and the narrow range of student demographics limited the overall generalizability of the study to the entire population of OT and PT students. Survey data collected was based on participant self-report, which potentially led to answers biased towards social norms. The collection of data in an anonymous online survey form could have improved the likelihood that participants responded candidly.

\section{Implications for Clinical Education}

Findings from this research could be used to help re-design CEE guidelines within educational programs in order to ensure maximal learning is achieved with each experience. Results could also help to mentor clinical educators in providing supportive knowledge, behaviors, and qualities that would ultimately allow students to have a more positive CEE. Overall awareness of the profound impact that belongingness has on students' learning is important for clinical educators to acknowledge. Without proper understanding of its importance, clinical instructors may inadvertently inhibit students from achieving their full potential.

Furthermore, the implications of these findings are important to clinical education program directors and clinical instructors alike. Education provided to CEE sites by directors of clinical education may emphasize the importance of creating environments in which students feel valued, significant, connected, and accepted. Clinical instructors should be encouraged to treat student therapists as young professionals and team members, rather than outsiders or underlings. Finally, creating a culture of inquisitiveness and exchange rather than acquiescence and delivery during the educational experience may promote student feelings of legitimacy and harmony conducive to learning and competence.

\section{CONCLUSION}

The analysis lends further support to the role that belongingness plays in advancing perceived competence during the CEE, extending similar research from the field of nursing to PT and OT professional students. The results suggest that the clinical education environment highly impacts student learning, especially in the area of belongingness.

\section{References}

American Occupational Therapy Association. (2002). Fieldwork performance evaluation for the occupational therapy student. Bethesda, MD: AOTA Press.

American Occupational Therapy Association. (2012). Fieldwork level II and occupational therapy students: A position paper. American Journal of Occupational Therapy, 66(Suppl. 6), S75-S77. https://doi.org/10.5014/ajot.2012.66S75 
American Occupational Therapy Association. (2015). Standards for continuing competence. American Journal of Occupational Therapy, 69(Suppl. 3), 6913410055. https://doi.org/10.5014/ajot.2015.696S16

American Physical Therapy Association. (2006). Physical Therapist Clinical Performance Instrument for Students [Press release]. Retrieved from https://cpi2.amsapps.com/docs/PT final revision 11-30-2010.pdf

Clarke, C., Martin, M., Sadlo, G., \& de-Visser, R. (2014). The development of an authentic professional identity on role-emerging placements. British Journal of Occupational Therapy, 77(5), 222-229. https://doi.org/10.4276/030802214x13990455043368

Competence. (2017). In Oxford Living Dictionaries. Retrieved from https://en.oxforddictionaries.com/definition/competence

Edgecombe, K., \& Bowden, M. (2009). The ongoing search for best practice in clinical teaching and learning: A model of nursing students' evolution to proficient novice registered nurses. Nurse Education in Practice, 9, 91-101. https://doi.org/10.1016/j.nepr.2008.10.006

Evenson, M., Barnes, M., \& Cohn, E. (2002). Brief report - Perceptions of level I and level II fieldwork in the same site. American Journal of Occupational Therapy, 56(1), 103-106. https://doi.org/10.5014/ajot.56.1.103

Grenier, M. L. (2015). Facilitators and barriers to learning in occupational therapy fieldwork education: Student perspectives. American Journal of Occupational Therapy, 69(Suppl. 2), 691218507. https://doi.org/10.5014/ajot.2015.015180

Grobecker, P. A. (2016). A sense of belonging and perceived stress among baccalaureate nursing students in clinical placements. Nurse Education Today, 36, 178-183. https://doi.org/10.1016/j.nedt.2015.09.015

Kim, M., \& Park, S. Y. (2011). Factors affecting the self-directed learning of students at clinical practice course for advanced practice nurse. Asian Nursing Research, 5(1), 48-59. https://doi.org/10.1016/S1976-1317(11)60013-3

Kim, Y. K., \& Sax, L. J. (2009). Student-faculty interaction in research universities: Differences by student gender, race, social class, and first-generation status. Research in Higher Education, 50, 437-459. https://doi.org/10.1007/s11162-009-9127-x

Levett-Jones, T., \& Lathlean, J. (2009). The ascent to competence conceptual framework: An outcome of a study of belongingness. Journal of Clinical Nursing, 18(20), 2870-2879. https://doi.org/10.1111/j.1365-2702.2008.02593.x

Levett-Jones, T., Lathlean, J., Higgins, I., \& McMillan, M. (2009). Staff-student relationships and their impact on nursing students' belongingness and learning. Journal of Advanced Nursing, 65(2), 316-324. https://doi.org/10.1111/j.1365-2648.2008.04865.x

Luanaigh, P. (2015). Becoming a professional: What is the influence of registered nurses on nursing students' learning in the clinical environment? Nurse Education Practice, 15(6), 450-456. https://doi.org/10.1016/j.nepr.2015.04.005 
Martin, M., Morris, J., Moore, A., Sadlo, G., \& Crouch, V. (2004). Evaluating practice education models in occupational therapy: Comparing 1:1, 2:1, and 3:1 placements. British Journal of Occupational Therapy, 67(5), 192-200. https://doi.org/10.1177/03080226040670052

Maslow, A. (1987). Motivation and Personality. New York NY: Harper and Row.

McCoy, M. A., Levett-Jones, T., \& Pitt, V. (2013). Development and psychometric testing of the Ascent to Competence Scale. Nurse Education Today, 33(1), 1523. https://doi.org/10.1016/..nedt.2011.11.003

Moss-Racusin, C. A., Dovidio, J. F., Brescoll, V. L., Graham, M. J., \& Handelsman, J. (2012). Science faculty's subtle gender biases favor male students. Proceedings of the National Academy of Science, 109, 16474-16479. https://doi.org/10.1073/pnas.1211286109

Nolan, C. A. (1998). Learning on clinical placement: The experience of six Australian student nurses. Nurse Education Today, 18(8), 622-629. https://doi.org/10.1016/S0260-6917(98)80059-2

Recker-Hughes, C., Wetherbee, E., Buccieri, K., FitzpatrickTimmerberg, J., \& Stolfi, A. (2014). Essential characteristics of quality clinical education experiences: Standards to facilitate student learning. Journal of Physical Therapy Education, 28(Suppl.1), 48-55. Retrieved from https://aptaeducation.org/members/jopte/index.cfm

Rindflesch, A., Hoversten, K., Patterson, B., Thomas, L., \& Dunfee, H. (2013). Students' description of factors contributing to a meaningful clinical experience in entrylevel physical therapist professional education. Work, 44(3), 265-274. https://doi.org/10.3233/WOR-121503

Russell, K., Alliex, S., \& Gluyas, H. (2016). Embracing the power of belongingness: A descriptive mixed method research study. Open Journal of Nursing, 6, 449-457. https://doi.org/10.4236?ojn.2016.66047

Thomson, R., Docherty, A., \& Duffy, R. (2017). Nursing students' experiences of mentorship in their final placement. British Journal of Nursing, 26(9), 514-521. https://doi.org.12968/bjon.2017.26.9.514 